\title{
A REMARK ON KÄHLER METRICS WITH CONICAL SINGULARITIES ALONG A SIMPLE NORMAL CROSSING DIVISOR
}

\author{
VED V. DATAR* ${ }^{*}$ JIAN SONG ${ }^{\dagger}$
}

\begin{abstract}
Recently it was shown by H. Guenancia and M. Păun that a singular metric satisfying the conical Kähler-Einstein equation with a simple normal crossing divisor is equivalent to a conical metric along that divisor. In this note, we present an alternative proof of their theorem.
\end{abstract}

Let $(X, \omega)$ be an $n$-dimensional Kähler manifold with a smooth Kähler metric $\omega$. We fix a divisor $D=\sum_{j=1}^{N}\left(1-\beta_{j}\right) D_{j}$, where $\beta_{j} \in(0,1)$ and $D_{j}$ 's are irreducible smooth divisors. We further assume that $D$ is a simple normal crossing divisor i.e for any $p \in \operatorname{Supp}(D)$ lying in the intersection of exactly $k$ divisors $D_{1}, \cdots, D_{k}$, there exists a coordinate chart $\left(U_{p},\left\{z_{j}\right\}\right)$ containing $p$, such that $\left.D_{j}\right|_{U_{p}}=\left\{z_{j}=0\right\}$ for $j=1, \cdots, k$.

If $s_{j}$ is the defining section of $D_{j}$ and $h_{j}$ is any smooth metric on the line bundle induced by $D_{j}$, then for sufficiently small $\epsilon_{j}>0, \theta_{j}=\omega+\epsilon_{j} \sqrt{-1} \partial \bar{\partial}\left|s_{j}\right|_{h_{j}}^{\beta_{j}}$ gives a Kähler metric on $X \backslash \operatorname{Supp}\left(D_{j}\right)$ with cone angle $2 \pi \beta_{j}$ along $D_{j}$. Now, set

$$
\theta=\sum_{j=1}^{N} \theta_{j}
$$

Then, $\theta$ is a smooth Kähler metric on $X \backslash S u p p(D)$. Moreover, for any $p \in \operatorname{Supp}(D)$ and any coordinate chart $\left(U_{p},\left\{z_{j}\right\}\right)$ as above, $\theta$ is uniformly equivalent to the standard cone metric

$$
\omega_{p}=\sum_{j=1}^{k} \frac{\sqrt{-1} d z_{j} \wedge d \bar{z}_{j}}{\left|z_{j}\right|^{2\left(1-\beta_{j}\right)}}+\sum_{j=k+1}^{N} \sqrt{-1} d z_{j} \wedge d \bar{z}_{j} .
$$

Next, for any $\lambda \in \mathbb{R}$, we consider the Monge-Ampère equation

$$
\left\{\begin{array}{l}
(\omega+\sqrt{-1} \partial \bar{\partial} \varphi)^{n}=\frac{e^{-\lambda \varphi} \Omega}{\prod_{j=1}^{N}\left|s_{j}\right|_{h_{j}}^{\left(1-\beta_{j}\right)}} \\
\omega_{\varphi}=\omega+\sqrt{-1} \partial \bar{\partial} \varphi>0
\end{array}\right.
$$

for some smooth volume form $\Omega$. By rescaling one can always assume that $\lambda=1,0,-1$. In the case that $\lambda=0$, we impose an additional normalization that $\sup _{M} \varphi=0$. The above equation arises when one considers the problem of prescribing the Ricci curvature of a conical metric and was first studied by Yau in [14. A natural question is whether conversely, any metric $\omega_{\varphi}$ solving (0.3) is in fact conical. The answer is provided by [6, 1, , 8] in the case of a smooth divisor and by [2, 7] in the general case. In the present note, we demonstrate that the situation with a simple normal crossing divisor is no harder than the one with a smooth divisor, thereby providing an alternate proof for the following theorem of H. Guenancia and M. Păun.

Research supported in part by National Science Foundation grants DMS-0847524 and the graduate fellowship of Rutgers University. 
Theorem 0.1. [7] If $\varphi$ is any bounded solution to (0.3), then there exists a constant $C>0$ such that

$$
C^{-1} \theta \leq \omega_{\varphi} \leq C \theta
$$

on $X \backslash \operatorname{Supp}(D)$, i.e., $\omega_{\varphi}$ is equivalent to a conical Kähler metric along $D$.

The theorem is essentially equivalent to certain second order estimates. We begin with

Lemma 0.1. There exists a constant $a>0$ such that, for any $j \in\{1, \cdots, N\}$

$$
\omega_{\varphi} \geq a \theta_{j}
$$

on $X \backslash \operatorname{Supp}(D)$.

Proof. We first assume that $\lambda=0$. The proof in the other two cases is similar (cf. Remark 0.1 ) with only a minor modification in the case of $\lambda=1$. We set $f_{j}=\log \left(\prod_{i=1, \ldots, N, i \neq j}\left|s_{i}\right|_{h_{i}}^{2\left(1-\beta_{i}\right)}\right)$. Then for some constant $A>>1, \sqrt{-1} \partial \partial f_{j}>-A \omega$ as currents. By Demailly's regularization theorem [5], there exist functions $F_{j, k} \in C^{\infty}(X)$ such that $F_{j, k} \searrow f_{j}$ and $\sqrt{-1} \partial \bar{\partial} F_{j, k}>-A \omega$. Now, consider the following family of Monge-Ampère equations

$$
\left\{\begin{array}{l}
\left(\omega+\sqrt{-1} \partial \bar{\partial} \varphi_{j, k}\right)^{n}=\frac{e^{\left(-F_{j, k}+c_{j, k}\right)} \Omega}{\left|s_{j}\right|_{h_{j}}^{\left(1-\beta_{j}\right)}} \\
\omega_{j, k}=\omega+\sqrt{-1} \partial \bar{\partial} \varphi_{j, k}>0 \\
\sup _{M} \varphi_{j, k}=0 .
\end{array}\right.
$$

It is well known [6, 1, 8, 3] that there always exists a solution $\varphi_{j, k}$ in $C^{2, \alpha, \beta_{j}}(X)$ for some $\alpha \in(0,1)$. Here $C^{2, \alpha, \beta}(X)$ are the Hölder spaces defined in [6]. Note that by integrating both sides of the equation, it is easy to see that the constants $c_{j, k}$ are uniformly bounded and converge to zero as $k \rightarrow \infty$. Since $\beta_{j} \in(0,1)$, from (0.6) it is clear that $\omega_{j, k}^{n} / \Omega \in L^{1+\epsilon}(X, \Omega)$ for some $\epsilon>0$ with uniform control over the $L^{1+\epsilon}$ norm. So, by Kolodziej's theorem [9], the solutions $\varphi_{j, k}$ are uniformly bounded in the $C^{0}$ norm. In fact, since from the equation $\omega_{j, k}^{n} / \Omega \rightarrow \omega_{\varphi}^{n} / \Omega$ in $L^{1}(X, \Omega)$, by the stability of solutions of complex Monge-Ampère equations [10], $\left|\varphi_{j, k}-\varphi\right|_{C^{0}(X)} \rightarrow 0$, as $k \rightarrow \infty$.

To obtain second order estimates, we note that $\operatorname{tr}_{\omega_{j, k}} \theta_{j}$ is bounded since $\varphi_{j, k} \in C^{2, \alpha, \beta_{j}}(X)$, and so for any $\delta>0$ and $B>0$, the quantity

$$
Q=\log \left(\left|s_{j}\right|_{h_{j}}^{2 \delta} \operatorname{tr}_{\omega_{j, k}} \theta_{j}\right)-B\left(\varphi_{j, k}-\epsilon_{j}\left|s_{j}\right|_{h_{j}}^{2 \beta_{j}}\right)
$$

attains its maximum value at some $p_{\max } \in X \backslash \operatorname{Supp}\left(D_{j}\right)$. Without loss of generality, we can assume that $\left|s_{j}\right|_{h_{j}} \leq 1$ on $X$. First, it follows from (0.6) and the fact that $\omega \leq c \theta$ for some $c>0$, that there exists a uniform $C>0$ such that $\operatorname{Ric}\left(\omega_{j, k}\right)>-C \theta_{j}$. Next, the bisectional curvature of $\theta_{j}$ is bounded above [8]. Hence by the Chern-Lu inequality [4, 11, 13], there exist constants $B, C>0$ independent of $j, k$ and $\delta$ such that

$$
\Delta_{\omega_{j, k}} Q \geq \operatorname{tr}_{\omega_{j, k}} \theta_{j}-C
$$

By the maximum principle and the uniform $C^{0}$ estimates, there exists an $a>0$ such that $\operatorname{tr}_{\omega_{j, k}} \theta_{j} \leq a^{-1} /\left|s_{j}\right|_{h_{j}}^{2 \delta}$ on $X$. Letting $\delta \rightarrow 0$,

$$
\omega_{j, k} \geq a \theta_{j} .
$$

Taking limit as $k \rightarrow \infty$ we prove that $\omega_{\varphi} \geq a \theta_{j}$ as currents. It follows, for instance from the regularization properties of Monge-Ampère flows [12], that $\omega_{\varphi}$ is in fact smooth away from $\operatorname{Supp}(D)$ and hence the inequality must be point-wise. This completes the proof of the lemma. 
Proof of Theorem 0.1. By adding the lower bounds from (0.5) for $j=1, \cdots, N$, we prove that there exists $C>0$ such that

$$
\omega_{\varphi} \geq C^{-1} \theta
$$

Since $\theta$ is locally equivalent to a cone metric with angle $2 \pi \beta_{j}$ along $D_{j}$, it is easy to check that

$$
\theta^{n}=\frac{\Omega^{\prime}}{\prod_{j=1}^{N}\left|s_{j}\right|_{h_{j}}^{2\left(1-\beta_{j}\right)}}
$$

for some continuous nowhere vanishing volume form $\Omega^{\prime}$, i.e., $\theta^{n}$ and $\omega_{\varphi}^{n}$ are uniformly equivalent on $X \backslash \operatorname{Supp}(D)$. Together with the lower bound on the metric, it directly gives the required upper bound on the metric.

Remark 0.1. The proof with $\lambda=-1$ can be carried out exactly as above. When $\lambda=1$, we approximate the solution $\varphi$ on the right hand side of (0.3) by quasi-plurisubharmonic functions as in [7] and again proceed as above.

\section{REFERENCES}

[1] Brendle, S. Ricci flat Kähler metrics with edge singularities, arXiv:1103.5454

[2] Campana, F., Guenancia, H. and Păun, M. Metrics with cone singularities along normal crossing divisors and holomorphic tensor fields, arXiv:1104.4879

[3] Chen, X., Donaldson, S. and Sun, S. Kähler-Einstein metrics on Fano manifolds, II: limits with cone angle less than $2 \pi$, arXiv:1212.4714

[4] Chern, S.S. On holomorphic mappings of Hermitian manifolds of the same dimension, Proc. Symp. Pure Math. 11, American Mathematical Society, 1968, pp. 157-170

[5] Demailly, J.P. Regularization of closed positive currents and intersection theory, J.Alg. Geom. 1 (1992), 361-409

[6] Donaldson, S.K. Kähler metrics with cone singularities along a divisor, Essays in mathematics and its applications, 49-79, Springer, Heidelberg, 2012

[7] Guenancia, H. and Păun, M. Conic singularities metrics with prescribed Ricci curvature: The case of general cone angles along normal crossing divisors, arXiv:1307.6375

[8] Jeffres, T., Mazzeo, R. and Rubinstein, Y. Kähler-Einstein metrics with edge singularities with appendix by Rubinstein, Y. and Li, C., arXiv:1105.5216

[9] Kolodziej, S. The complex Monge-Ampère equation, Acta Math. 180 (1998),69-117

[10] Kolodziej, S. The Monge-Ampère equation on compact Kähler manifolds, Indiana Univ. Math. J. 52 (2003), no. 3, 667-686

[11] Lu, Y.C. Holomorphic mappings of complex manifolds, J. Diff. Geom. 2 (1968), 299-312

[12] Song, J. and Tian, G. The Kähler-Ricci flow through singularities, arXiv0909.4898

[13] Yau, S.-T. A general Schwarz lemma for Kähler manifolds, Amer. J. Math. 100 (1978), 197-203

[14] Yau, S.-T. On the Ricci curvature of a compact Kähler manifold and the complex Monge-Ampère equation, I, Comm. Pure Appl. Math. 31 (1978), no. 3, 339-411

* Department of Mathematics, Rutgers University, Piscataway, NJ 08854

E-mail address: veddatar@math.rutgers.edu

$\dagger$ Department of Mathematics, Rutgers University, Piscataway, NJ 08854

E-mail address: jiansong@math.rutgers.edu 